\title{
Espécies raras de Phallales (Agaricomycetidae, Basidiomycetes) no Nordeste do Brasil ${ }^{1}$
}

\author{
Anileide Gomes Leite ${ }^{1}$, Bianca Denise Barbosa Silva ${ }^{1}$, Ricardo Souza Araújo² e Iuri Goulart Baseia ${ }^{1,3}$
}

Recebido em 6/02/2006. Aceito em 10/08/2006

\begin{abstract}
RESUMO - (Espécies raras de Phallales (Agaricomycetidae, Basidiomycetes) no Nordeste do Brasil). Seis espécies interessantes de Phallales são registradas para a Mata Atlântica: Aseroëfloriformis Baseia \& Calonge, Geastrum setiferum Baseia, Ileodictyon cibarium Tulasne ex Raoul, Laternea triscapa Turpin, Phallus pygmaeus Baseia e Staheliomyces cinctus E. Fischer. Adicionalmente, são fornecidas chave de identificação e comentários sobre a taxonomia e ecologia dessas espécies.
\end{abstract}

Palavras-chave: Basidiomycota, Gasteromycetes, MEV, taxonomia, neotrópicos

\begin{abstract}
Rare species of Phallales (Agaricomycetidae, Basidiomycetes) in Northeast Brazil). Six interesting species of Phallales are recorded from the Atlantic rainforest: Aseroëfloriformis Baseia \& Calonge, Geastrum setiferum Baseia, Ileodictyon cibarium Tulasne ex Raoul, Laternea triscapa Turpin, Phallus pygmaeus Baseia and Staheliomyces cinctus E. Fischer. A key and comments on taxonomy and ecology of these species are also provided.
\end{abstract}

Key words: Basidiomycota, Gasteromycetes, SEM, taxonomy, neotropics

\section{Introdução}

A ordem Phallales foi estabelecida por Fischer (1885), e atualmente compreende cinco famílias, cerca de 60 gêneros e 330 espécies (Kirk et al. 2001). Seus representantes apresentam ampla distribuição geográfica, embora, a maior diversidade provavelmente esteja nas florestas tropicais (Hawksworth 2001), onde são importantes na reciclagem da matéria orgânica vegetal. Ainda possuem interessantes estratégias na dispersão dos esporos, utilizando, na maioria das vezes, insetos como agentes dispersores (Oliveira \& Morato 2000; Shaw \& Roberts 2002).

Na última década, a estimativa da diversidade dos fungos foi amplamente discutida (Hawksworth 1993; Hyde 1997) e hoje sabe-se que os fungos são, provavelmente, o segundo grupo com maior riqueza de espécies, atrás apenas dos insetos (Hyde \& Hawksworth 1997). Até o momento, foram descritas 72.000 espécies de fungos, porém estima-se que haja pelo menos 1,5 milhões de espécies no mundo
(Hawksworth 2001), ou seja, apenas cerca de 5\% da diversidade estimada é conhecida. Neste contexto, objetiva-se contribuir para o conhecimento dessa diversidade ainda desconhecida, enfatizando a taxonomia e os aspectos ecológicos de representantes raros de Phallales. Apesar do pouco conhecimento deste grupo de fungos no nordeste do Brasil (Baseia \& Milanez 2002; Baseia et al. 2003a; b; Baseia \& Calonge 2005), várias espécies novas destes fungos foram descritas para os neotrópicos nos últimos anos (Calonge \& Mata 2004; 2005; Calonge et al. 2005a; b) e, de acordo com Jorge E. Wright (comunicação pessoal em 2003), o Brasil teria muitas espécies ainda desconhecidas.

\section{Material e métodos}

As coletas do material foram realizadas durante 0 período chuvoso de janeiro/2003 a junho/2004, na Reserva Biológica (REBIO) Guaribas (06 $45^{\circ}$ 'S $35^{\circ} 12^{\prime}$ W) no Estado da Paraíba, na REBIO de Serra

\footnotetext{
1 Universidade Federal do Rio Grande do Norte, CB, Departamento de Botânica, Ecologia e Zoologia, Laboratório de Micologia, Campus Universitário, Lagoa Nova, 59072-970 Natal, RN, Brasil

2 Universidade Estadual Vale do Acaraú, Laboratório de Sistemática Vegetal, Rua Francisco Batista Leal 505, Centro, 62320-000 Tianguá, CE, Brasil

3 Autor para correspondência: baseia@cb.ufrn.br
} 
Negra (08 $\left.38^{\circ} \mathrm{S}-38^{\circ} 02^{\prime} \mathrm{O}\right)$ e na Reserva Ecológica (RESEC) de Gurjaú (08⒉'S - 31 $56^{\circ}$ 'O), no Estado de Pernambuco. Foram também procedidas coletas no período chuvoso de julho/2004 a março/2005 no Parque Estadual Dunas do Natal $\left(05^{\circ} 46^{\prime} \mathrm{S}-35^{\circ} 12^{\prime} \mathrm{O}\right)$ e na Reserva Particular do Patrimônio Natural (RPPN) Mata Estrela ( $\left.06^{\circ} 22^{\prime} \mathrm{S}-35^{\circ} 00^{\prime} \mathrm{O}\right)$, ambos localizados no Estado do Rio Grande do Norte. Adicionalmente, foi efetuada coleta em março/2005, na mata "Cachoeira da Fumaça", no município de Viçosa do
Ceará ao norte do Estado do Ceará (033's $\left.41^{\circ} 05^{\prime} \mathrm{W}\right)$.

A análise do material seguiu as técnicas rotineiras empregadas em estudos taxonômicos de gasteromicetos (Miller \& Miller 1988). A terminologia micológica segue a nomenclatura proposta por Kirk et al. (2001). A padronização das cores segue Kornerup \& Wanscher (1978). Os esporos foram examinados em Microscópio Eletrônico de Varredura (MEV) modelo Phillips XL 20.

\section{Resultados e discussão}

Chave para identificação das espécies de Phallales estudadas

1. Gleba pulverulenta, deiscência regular com exoperídio em forma de estrela 2. Geastrum setiferum

1. Gleba gelatinosa, deiscência irregular, nunca em forma de estrela 2

2. Receptáculo com três arcos persistentes 4. Laternea triscapa

2. Receptáculo sem arcos 3

3. Ápice do receptáculo campanulado 5. Phallus pygmaeus

3. Ápice do receptáculo não campanulado 4

4. Receptáculo com braços vestigiais em forma de girassol. 1. Aseroë floriformis

4. Receptáculo cilíndrico ou em forma de rede 5

5. Gleba presente em vários peridíolos, odor fétido 3. Ileodictyon cibarium

5. Gleba em forma de colar pouco abaixo do ápice, odor agradável 6. Staheliomyces cinctus

Aserö̈ floriformis Baseia \& Calonge, Mycotaxon 92: 169-172, 2005.

Fig. 1

Ovo globoso a subgloboso, 15-20 mm diâm., epígeo, branco amarelado (KW 4A3), deiscência irregular; estipe cilíndrico, esponjoso, avermelhado (KW 11A6) a rosa (KW 11A4), 30-40 mm alt., 5-8 mm larg.; receptáculo em forma de girassol, 15-35 mm diâm., ramificações ausentes, rosa (KW 11A4); disco central perfurado e avermelhado nas bordas (KW 11 A8), coberto por uma gleba mucilaginosa, cinza amarronzada (KW 11F1), odor desagradável; esporos cilíndricos a baciliformes, 4-6×1,5-2 $\mu \mathrm{m}$, lisos, hialinos.

Material examinado: BRASIL. Rio Grande do Norte: Natal, Parque Estadual Dunas do Natal, 25/VI/2005, Baseia 1545 (UFRN-Fungos 193; URM 77091; MA-Fungi 59460); Baía Formosa, RPPN Mata Estrela, 25/VI/2005, Baseia 1548 (UFRN-Fungos 193). Ceará: Viçosa do Ceará, Cachoeira da Fumaça, 3/III/2005, Araújo 046 (UFRN-Fungos 207).

Comentários: Aseroë possui apenas três espécies: A. rubra Labill., A. arachnoidea E. Fischer e A. floriformis Baseia \& Calonge, esta última recen- temente descoberta em vegetação de Mata Atlântica no Estado do Rio Grande do Norte (Baseia \& Calonge 2005). Esse gênero pode ser caracterizado pelo seu basidioma, composto por um receptáculo e um estipe cilíndrico bem diferenciado que emerge de uma volva esbranquiçada. Com exceção de A. floriformis, as demais espécies apresentam várias ramificações que se originam a partir da margem do receptáculo. Esse gênero apresenta distribuição pantropical (Dring 1980),
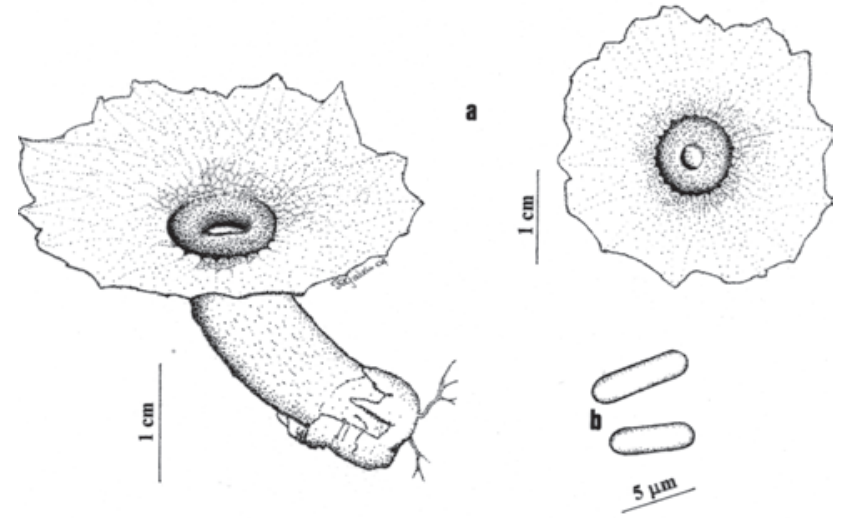

Figura 1. Aseroë floriformis Baseia \& Calonge: a. Basidiomas. b. Basidiósporos. 
contudo A. rubra já foi registrada para a Europa (Spooner 1994). No Brasil, temos registro de A. floriformis apenas para o Estado do Rio Grande do Norte (Baseia \& Calonge 2005; Baseia et al. 2006) e Ceará. A grande variabilidade na morfologia dos basidiomas tem induzido vários autores a criar sinônimos, especialmente no caso de $A$. rubra, com 25 nomes publicados como espécies distintas e que, segundo Dring (1980), na verdade não passam de sinônimos. Uma característica ecológica interessante é a gleba desta espécie, quando madura, exalar odor de fezes de bovinos, atraindo insetos. Nos trabalhos de campo, foram observados vários tipos de insetos sobre os basidiomas de $A$. floriformis, principalmente moscas e besouros.

Geastrum setiferum Baseia, Mycotaxon 84: 135-139, 2002.

Fig. 2

Ovo globoso a subgloboso, 1,5-2,3 cm diâm., epígeo, marrom escuro (KW 7F5), deiscência regular; exoperídio quando aberto medindo $3-5 \mathrm{~cm}$ larg., composto por 5-8 raios pontiagudos e recurvados; camada micelial escamosa; endoperídio laranja acinzentado (KW 5B3), séssil, globoso, 10-15 mm diâm., superfície recoberta por setas cimbiformes (90-120 $\times 20-30 \mu \mathrm{m})$, marrons; peristômio definido, sulcado tornando-se fibriloso nas bordas; columela distinta e fusiforme; esporos globosos, 2,5-3 $\mu \mathrm{m}$, verrugosos, amarronzados; capilícios longos e sem ramificações, 3-4 $\mu$ m diâm., amarronzados, podendo apresentar incrustações na superfície.

Material examinado: BRASIL. Pernambuco: Serra Negra, REBIO de Serra Negra, 23/V/2003, Baseia 1316 (UFRN-Fungos 195; URM 77072, 77077). Paraíba: Mamanguape, REBIO Guaribas, 12/VI/2003, Baseia 1367 (UFRN-Fungos 206).

Comentários: diante das novas técnicas empregadas na taxonomia moderna, como dados ultraestruturais e moleculares, o posicionamento e a hierarquia de diversos grupos de fungos foram profundamente afetados. A família Geastraceae que até pouco tempo pertencia à ordem Lycoperdales, é um exemplo claro desta alteração, com a invalidação de Lycoperdales (Krüger et al. 2001). Geastraceae migrou para Phallales, com base em dados moleculares que indicam a proximidade evolutiva entre Geastraceae e Phallaceae (Hibbett et al. 1997, Hibbett \& Donoghue 1998). Geastrum setiferum se distingue das demais espécies do gênero por apresentar o endoperídio recoberto por setas minúsculas (Fig. 2b). Ao contrário

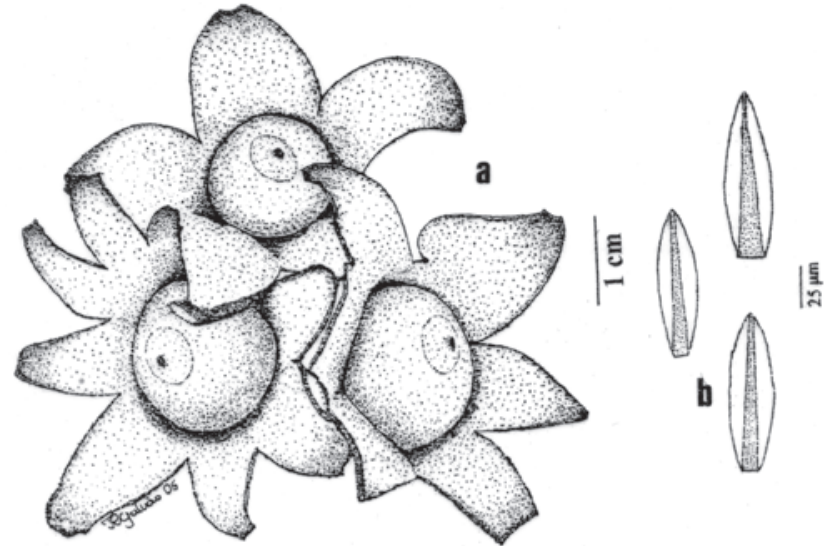

Figura 2. Geastrum setiferum Baseia: a. Basidiomas. b. Setas do endoperídio.

das demais espécies aqui estudadas, os esporos de G. setiferum são globosos e ornamentados (Fig. 7a). Para descrição completa, consultar Baseia \& Milanez (2002).

Ileodictyon cibarium Tulasne ex Raoul, Ann. Sci Nat. Bot. Ser. 3, 2: 114 (1844).

Fig. 3

Ovo subgloboso, 20-25 mm diâm., epigeo, branco amarelado (KW 4A2) a branco (KW 4A1), deiscência irregular; receptáculo expandido com 70-80× 70-80 mm, branco (KW 4A1), formado por vários arcos que formam uma rede globosa de pentágonos e hexágonos, séssil; diversas glebas (12-16) originadas na porção interna dos arcos, mucilaginosas, odor desagradável; esporos elípticos, 3,5-4 ×1,5-2 $\mu \mathrm{m}$, lisos, hialinos.

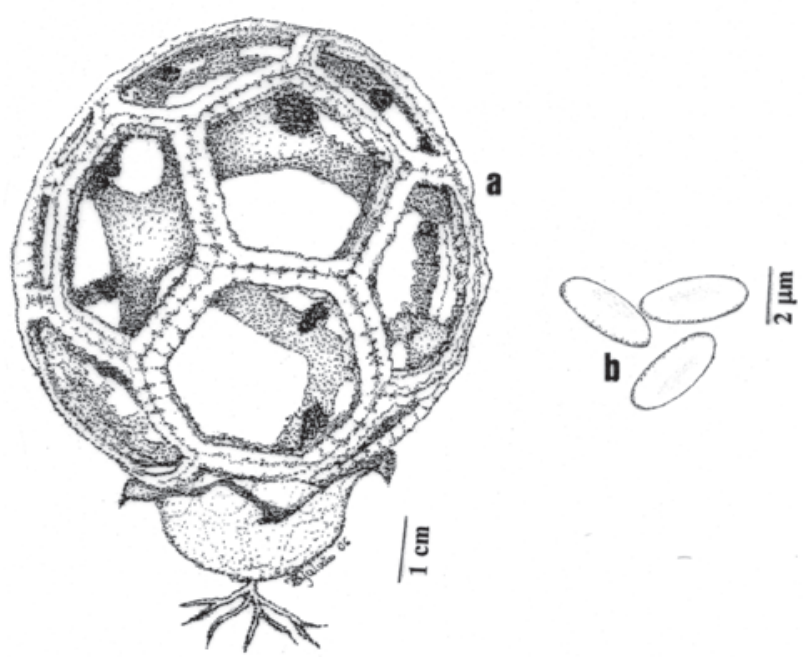

Figura 3. Ileodictyon cibarium Tulasne ex Raoul: a. Basidioma. b. Basidiósporos. 
Material examinado: BRASIL. Rio Grande do Norte: Natal, Parque Estadual Dunas do Natal, 25/V/2005, Baseia 1521 (UFRN-Fungos 196).

Comentários: este taxon apresenta uma morfologia notável, em que o receptáculo forma uma estrutura poliédrica denominada fulereno, uma forma alotrópica do carbono, semelhante a uma bola de futebol. Segundo Gooday \& Zerning (1997) esta morfologia confere a Ileodictyon cibarium uma área maior para que os agentes dispersores, moscas na maioria das vezes, sejam atraídos pelo cheiro desagradável das inúmeras glebas presentes no interior do receptáculo. Através de observações dos esporos em MEV, foi constatado que os esporos são lisos e elípticos com uma pequena depressão na porção central (Fig. 7b). Essa morfologia, provavelmente, favorece sua ingestão por insetos, e a presença de uma substância adesiva recobrindo a superfície possibilita a dispersão dos esporos pela impregnação no corpo desses insetos (Shaw \& Roberts 2002).

Laternea triscapa Turpin, Dict. Sci Nat. 2 ed. 25: 248, 1822.

Fig. 4

Ovo subgloboso, 20-25 mm diâm., epígeo, branco amarelado (KW 4A2), deiscência irregular; receptáculo obovóide, até $60 \times 20 \mathrm{~mm}$, alaranjado (KW 5A6), apresentando três arcos livres na base e unidos no ápice; gleba mucilaginosa presente na porção superior interna dos arcos, marrom amarelada (KW 5F6), odor desagradável; esporos cilíndricos a elípticos, 3,5-4× 2-2,5 $\mu \mathrm{m}$, lisos, hialinos.

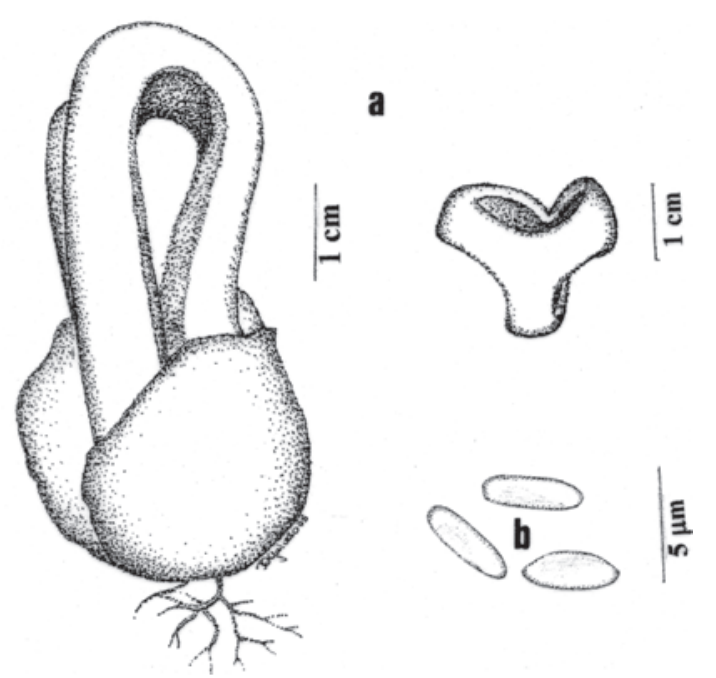

Figura 4. Laternea triscapa Turpin: a. Basidioma. b. Basidiósporos.
Material examinado: BRASIL. Rio Grande do Norte: Natal, Parque Estadual Dunas do Natal, 12/VII/2004, Baseia 1453 (UFRN-Fungos 194).

Comentários: esta espécie é muito próxima de Laternea pusilla Berk. \& M.A. Curtis, caracterizada por basidiomas maiores $(70 \times 30$ milímetros $)$ com três a quatro colunas ornamentadas, enquanto em $L$. triscapa o basidioma é menor e falta qualquer tipo da ornamentação (Calonge et al. 2005b). De acordo com Dring (1980), Clathrus brasiliensis, descrita por Fischer em 1886 para o Brasil, é um sinônimo desta espécie.

Phallus pygmaeus Baseia, Mycotaxon 85: 77-79, 2003b.

Fig. 5

Ovo globoso a subgloboso, 20-30 mm diâm., epígeo, marrom oliva (KW 4F4) no ápice, branco amarelado (KW 4A2) na porção inferior, deiscência irregular; estipe cilíndrico, reticulado, despigmentado, $5-10 \mathrm{~mm}$ alt., 1-2 mm diâm.; píleo campanulado, superfície lisa, ápice perfurado; gleba olivácea (KW 1F5), fétida, mucilaginosa, esporos elípticos, 3-4×1-1,5 $\mu \mathrm{m}$, lisos, hialinos.

Material examinado: BRASIL. Pernambuco: Cabo de Santo Agostinho, RESC de Gurjaú, 9/IV/2003, Baseia 1294 (UFRN-Fungos 199; URM 77078, 77079).

Comentários: Phallus pygmaeus pode ser facilmente reconhecido pelo tamanho diminuto das frutificações que não excedem $1 \mathrm{~cm}$ de altura, bem como pela superfície lisa do píleo e pelo hábito lignícola,

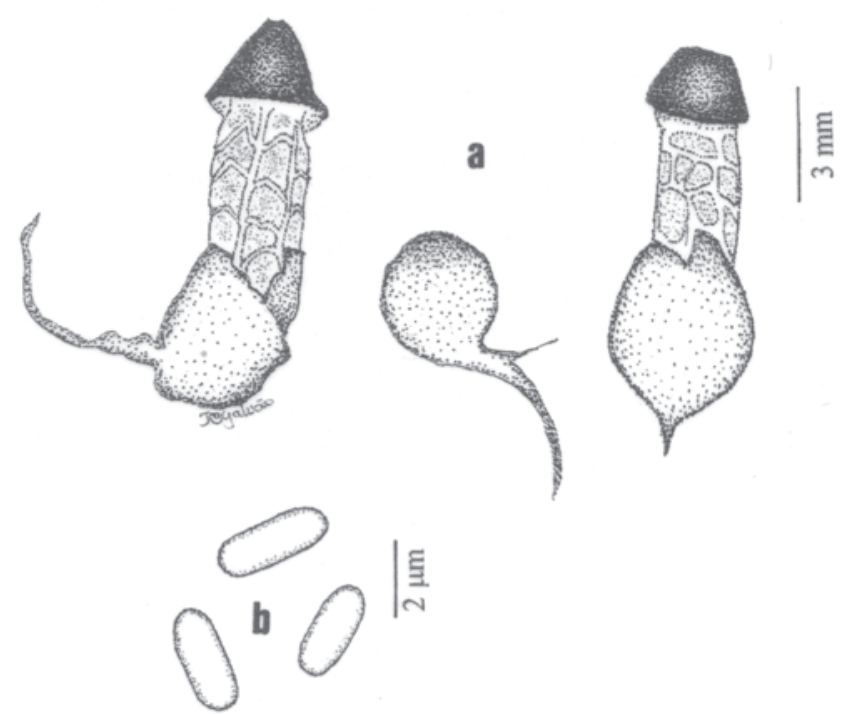

Figura 5. Phallus pygmaeus Baseia: a. Basidioma. b. Basidiósporos. 
característica ecológica peculiar. Como nas demais espécies deste gênero, a gleba, quando madura, exala odor de carniça, o que atrai vários insetos. Nos trabalhos de campo foram observadas formigas e pequenas moscas sobre os basidiomas. Para descrição completa, ver Baseia et al. (2003).

Staheliomyces cinctus E. Fischer, Mitt. Naturf. Ges. Bern 35: 142 (1920-1921).

Fig. 6

Ovo oval, 15-20×12-16 mm, epígeo, marrom oliva (KW 4F6), deiscência irregular; estipe cilíndrico e perfurado, branco amarelado (KW 5A1), $100 \times 140 \mathrm{~mm}$ de altura; gleba marrom amarelada (KW 5E6) formando uma banda constrita em torno da porção superior do receptáculo, odor agradável, mucilaginosa, esporos elípticos, 2,5-3×1,2×1,5 $\mu \mathrm{m}$, lisos, hialinos.

Material examinado: Brazil. Rio Grande do Norte: Natal, Parque Estadual Dunas do Natal, 16/IV/2004, Baseia 1364 (UFRN-Fungos 123).

Comentários: Staheliomyces cinctus tem ocorrência apenas para os neotrópicos, sendo de fácil

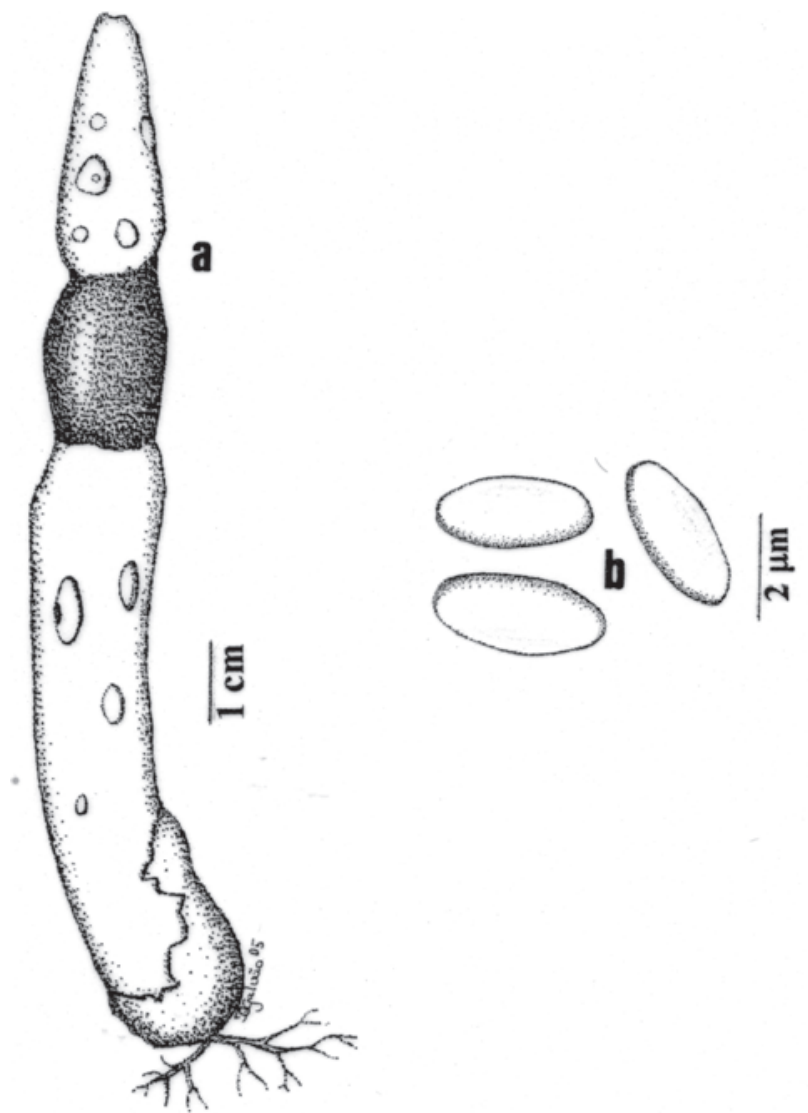

Figura 6. Staheliomyces cinctus E. Fischer: a. Basidioma. b. Basidiósporos.
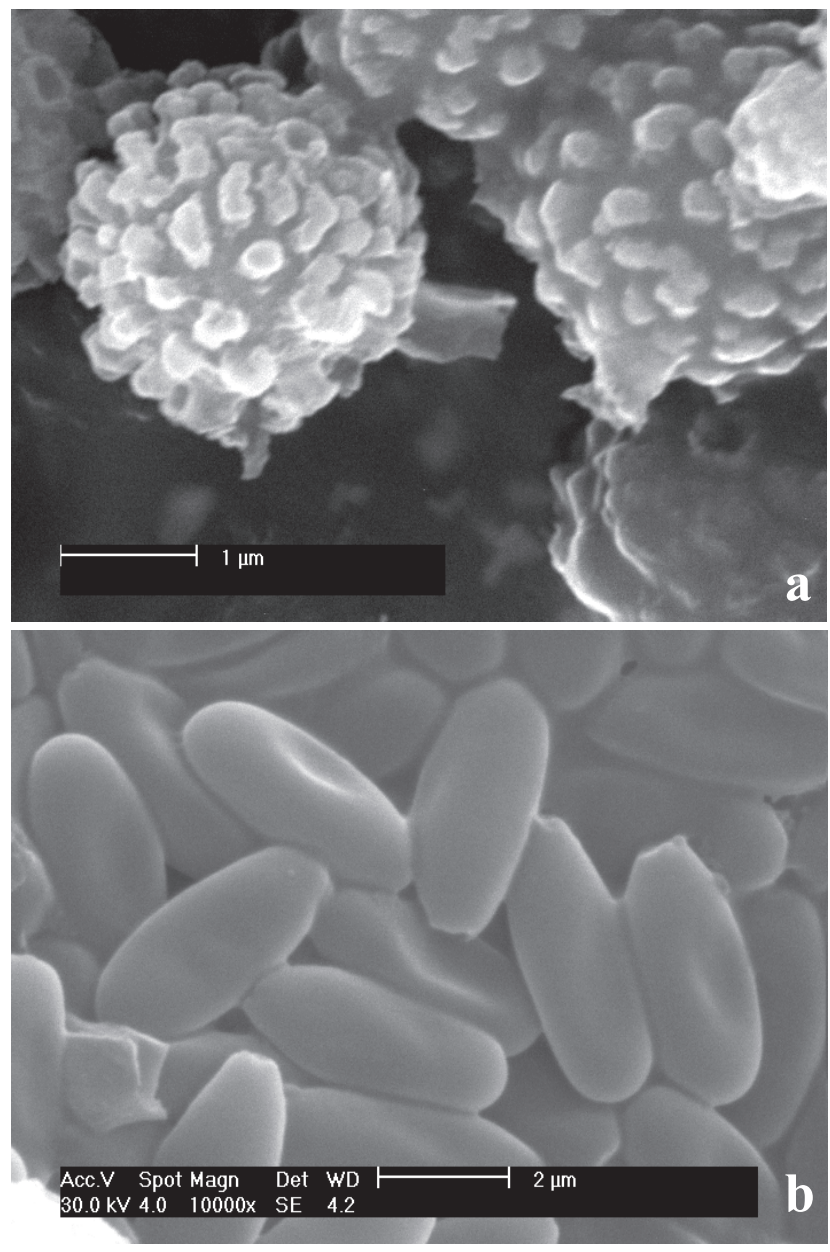

Figura 7. Basidiósporos em MEV: a. Geastrum setiferum. b. Ileodictyon cibarium.

identificação devido à morfologia singular. Esse gênero é representado por apenas uma espécie que lembra um Phallus, mas difere pela presença de uma forte constrição na porção superior média do pseudoestipe, recordando um cinto, onde a gleba é encontrada. Esta espécie foi registrada apenas para o Suriname, Guiana, Bolívia, Costa Rica, Equador, Panamá e Peru (Dennis 1970; Calonge et al. 2005b).

\section{Agradecimentos}

Os autores agradecem aos Professores Hanns Kreisel (Universität Greifswald, Alemanha) e Francisco de Diego Calonge (Real Jardín Botánico de Madrid, Espanha), pela leitura crítica do manuscrito. Agradecimentos ainda são devidos a Tereza Cristina de Oliveira Galvão, pelas ilustrações; ao Conselho Nacional de Desenvolvimento Científico e Tecnológico (CNPq), pelo suporte financeiro. 


\section{Referências}

Baseia, I.G.; Cortez, V.G. \& Calonge, F.D. 2006. Rick species revision: Mitremyces zanchianus versus Calostoma zanchianum. Mycotaxon 95: 113-116.

Baseia, I.G. \& Calonge, F.D. 2005. Aseroë floriformis, a new phalloid with a sunflower shaped receptacle. Mycotaxon 92: 169-172.

Baseia I.G.; Cavalcanti M.A.\& Milanez A.I. 2003a. Additions to our knowledge of the genus Geastrum (Phallales: Geastraceae) in Brazil. Mycotaxon 85: 409-416.

Baseia, I.G.; Gibertoni, T.B. \& Maia, L.C. 2003b. Phallus pygmaeus, a new minute species from a Brazilian tropical rainforest. Mycotaxon 85: 77-79.

Baseia, I.G. \& Milanez, A.I. 2002. Geastrum setiferum (Gasteromycetes): a new species with a setose endoperidium. Mycotaxon 84: 135-139.

Calonge, F.D. \& Mata, M. 2004. A new species of Geastrum from Costa Rica and México. Boletín de la Sociedad Micologica de Madrid 28: 331-335.

Calonge, F.D. \& Mata, M. 2005. Crucibulum laeve var. magnum var. nov. y Arcangeliella scissilis, encontrados en Costa Rica. Boletín de la Sociedad Micologica de Madrid 29: 43-48.

Calonge, F.D.; Kreisel, H. \& Mata, M. 2005a. Phallus atrovolvatus, a new species from Costa Rica. Boletín de la Sociedad Micologica de Madrid 29: 5-8.

Calonge, F.D.; Mata, M. \& Carranza, J. 2005b. Contribición al catálogo de los Gasteromycetes (Basidiomycotina, Fungi) de Costa Rica. Anales del Jardín Botánico de Madrid 62(1): 23-45.

Dennis, R.W.G. 1970. Fungus flora of Venezuela and adjacent countries. Kew Bulletin Additional Series 3, London, J. Cramer.

Dring, D.M. 1980. Contributions towards a rational arrangement of the Clathraceae. Kew Bulletin 35: $1-96$.

Fischer, E. 1885. Zur Entwicklungsgeschichte der Fruchtkörper einiger Phalloideen. Annales du Jardin Botanique de Buitenzorg 6: 1-51.
Gooday, G.W. \& Zerling, J. 1997. Ileodictyon cibarium: the basket fungus as a buckyball. Mycologist 11(4): 184-186.

Hawksworth, D.L. 2001. The magnitude of fungal diversity: the 1,5 million species estimate revised. Mycological Research 105(12): 1422-1432.

Hawksworth, D.L. 1993. The tropical fungal biota: census, pertinence, prophylaxis and prognosis. Pp. 265-293. In: S. Issac; J.C. Frankland; R. Watling \& A.J.S Whalley (eds.). Aspects of Tropical Mycology. Cambridge, Cambridge University Press.

Hibbett, D.S. \& Donoghue, M.J. 1998. Integrating phylogenetic analysis and classification in fungi. Mycologia 90: 347-356.

Hibbett, D.S.; Pine, E.M.; Langer, E.; Langer, G. \& Donoghue, M.J. 1997. Evolution of gilled mushrooms and puffballs inferred from ribossomal DNA sequences. Proceeding of the National Academy of Science 94: 12002-12006.

Hyde, K.D. 1997. Can we rapidly measure fungal diversity? Mycologist 11(4): 176-178.

Hyde, K.D. \& Hawksworth, D.L. 1997. Measuring and monitoring the biodiversity of microfungi. Pp:141-156. In: K. D. Hyde (ed.). Biodiversity of tropical microfungi. Hong Kong, Hong Kong University Press.

Kirk, P.M.; Cannon, P.F.; David, J.C. \& Stalpers, J.A. 2001. Ainsworth \& Bisby's Dictionary of the Fungi. $9^{\text {th }}$ edition, Wallingford, CABI Int. Publ.

Kornerup, A. \& Wanscher, J.H. 1978. Methuen handbook of colours. $3^{\text {rd }}$ edition. London, Eyre Methuen.

Krüger, D.; Binder, M.; Fischer, M. \& Kreisel, H. 2001. The Lycoperdales. A molecular approach to the systematic of some gasteroid mushrooms. Mycologia 93(6): 947-957.

Miller, O.K. Jr. \& Miller, H.H. 1988. Gasteromycetes: Morphology and Developmental Features. Eureka, Mad River.

Oliveira, M.L. \& Morato, E.F. 2000. Stingless bees (Hymenoptera, Meliponini) feeding on stinkhorn spores (Fungi, Phallales): robbery or dispersal? Revista Brasileira de Zoologia 17(3): 881-884.

Shaw, D.E. \& Roberts, P. 2002. Bees and phalloid exudate. Mycologist 16(3): 109.

Spooner B. 1994. Aseroe rubra at Oxshott. Mycologist 8: $1-153$. 